\title{
Recent Advances in Directed Assembly of Nanowires or Nanotubes
}

\author{
Mei Liu ${ }^{1,2 *}$, Zhizheng $\mathrm{Wu}^{1}$, Woon Ming Lau ${ }^{3}$, Jun Yang ${ }^{4 *}$
}

(Received 9 September 2012; accepted 12 September 2012; published online 20 September 2012.)

\begin{abstract}
Nanowires and nanotubes of diverse material compositions, properties and/or functions have been produced or fabricated through various bottom-up or top-down approaches. These nanowires or nanotubes have also been utilized as potential building blocks for functional nanodevices. The key for the integration of those nanowire or nanotube based devices is to assemble these one dimensional nanomaterials to specific locations using techniques that are highly controllable and scalable. Ideally such techniques should enable assembly of highly uniform nanowire/nanotube arrays with precise control of density, location, dimension or even material type of nanowire/nanotube. Numerous assembly techniques are being developed that can quickly align and assemble large quantities of one type or multiple types of nanowires through parallel processes, including flow-assisted alignment, Langmuir-Blodgett assembly, bubble-blown technique, electric/magnetic- field directed assembly, contact/roll printing, knocking-down, etc.. With these assembling techniques, applications of nanowire/nanotube based devices such as flexible electronics and sensors have been demonstrated. This paper delivers an overall review of directed nanowire assembling approaches and analyzes advantages and limitations of each method. The future research directions have also been discussed.
\end{abstract}

Keywords: Keywords: Nanowire; Nanotube; Assembly; Shear force; Contact printing; Dielectrophoresis; Microfluidics; Nanosystems; Nanodevices

Citation: Mei Liu, Zhizheng Wu, Woon Ming Lau and Jun Yang, "Recent Advances in Directed Assembly of Nanowires or Nanotubes", Nano-Micro Lett. 4 (3), 142-153 (2012). http://dx.doi.org/10.3786/nml.v4i3. p142-153

\section{Introduction}

One dimensional nanomaterials such as nanowires (NWs) and nanotubes (NTs) can now be synthesized with bottom-up approaches with high-throughput and controllable material properties, such as their shape, dimension, atomic composition and doping concentration. Also, a wide range of those NW/NT-based devices such as field effect transistors, single virus detectors, photo-detectors, and chemical/biological sensors have been demonstrated with superior performances.

Briefly, NW/NT devices are usually fabricated with such a process: NW/NTs are firstly synthesized with a particular process, and dispersed onto a device substrate under some specific driving mechanism, with those driving mechanisms the NWs will align, where the orientations and interspacing of NWs over large area is essential parameters for nanodevices with logic functions. Thereafter, a photo- or electron-beam lithography process is applied to pattern metallic electrodes to the NWs, thus the NWs can be integrated into electronic nanodevices. As we see, the assembly technique is one of the keys for the fabrication of powerful nanodevices. Since reliability, cost and yield determine

\footnotetext{
${ }^{1}$ School of Mechatronics Engineering and Automation, Shanghai University, Shanghai 200072, China

${ }^{2}$ State Key Laboratory of Transducer Technology, Shanghai Institute of Microsystem and Information Technology, Chinese Academy of Sciences, Shanghai 200050, China

${ }^{3}$ Green Energy and Green Manufacturing Technology R\&D Center, Chengdu 610207, China

${ }^{4}$ Department of Mechanical and Materials Engineering, the University of West ern Ontario, London, Ontario, Canada, N6A 5B9

*Corresponding author. E-mail: mliu@shu.edu.cn, jyang@eng.uwo.ca
} 
commercialization of NW/NT based nanodevices, a reliable, low-cost and efficient assembly method, which is capable of NW assembly with reasonable alignment, density, uniformity and even controllable types, is essential for the prosperity of nanodevices.

Till now, researchers have developed a variety of NW/NT assembly methods on various substrates, which can generally be categorized as two strategies [1-9]: (1) Alignment-after-Growth, which means, the NWs are assembled on substrates after synthesis; (2) Alignment-during-growth, which means, the NWs are assembled and aligned directly during the synthesis process. For example, by pre-defining the locations of catalyst and growth space of the NWs [10-13], applying electric field in the seeded growth system [14-15], applying directional air flow in the growth system [1617], or directly on a surface based on physical atomic structures [18-28], by electrospinning [29-31], or superaligning [32-33], NWs can be aligned as grown simultaneously. However, these approaches are limited to a few types of materials and relatively simple structures, and the uniformity of the grown NWs is hard to control. While the Alignment-after-Growth approaches can manipulate NWs as individual structures and assemble it into complex architectures, and put no limitations on NW materials, so are more flexible and recommendable for nanodevices.

In this review article, we will summarize the stateof-art research progress on the first strategy mentioned above: Alignment-after-Growth assembly techniques. Basically there are several factors for evaluating an assembly technique: simplicity, reliability, cost, yield, its limitation on NW material, applicability to flexible substrates, ability to assemble multiple types of NWs, etc. Each method will be discussed based on those factors, and the future research efforts will be recommended.

\section{Assembly-After-Growth approaches}

Most alignment-after-growth approaches are carried out in a specific solution, and during the assembly process the fluid acts as lubricant. The NWs are mobile in the assembly system, and the assembly processes take place on smooth substrate surfaces. Generally, NWs after some particular treatment can be uniformly dispersed in a solution. The concentration of NWs could be readily adjusted through condensation or dilution. If the NW suspension is cast onto a substrate, some NWs will settle on the substrate surface randomly. The adhering strength between the NWs and the substrate is determined by the interaction between them, and can be enhanced through means like van der Waals forces or hydrogen bonding interactions. Meanwhile, if some type of driving mechanism is added into the assembly process, for example, shear forces, or electric forces, the depositing NWs will adopt the orientation of the external force and become aligned while residing onto the substrate.

\section{Intermolecular-Force-Driven assembly}

Intermolecular-force could be applied to intensify the adhesion between the assembled NWs and the substrate. Two common techniques including the hybridization of deoxyribonucleic acid (DNA), and protein-protein interactions to form protein complexes [34-37] have been used. In either way, complimentary structures are linked to the NWs and the substrate respectively, and the NWs are bound to the substrate through the complex formed when the two complementary surfaces are brought into contact in the assembly solution. For example, a single-stranded DNA can bind selectively to the complementary DNA strand, which can direct the assembly of nanostructures [34-36].

Biorecognition is an effective means to drive the assembly of NW/NTs by intermolecular-forces between complementary biomolecules. It is simple and can be easily adapted to many types of materials, but is difficult for this technique to direct the alignment of the $\mathrm{NW}$ /NTs during the assembly process, which means, assembled NW/NTs may exhibit random directions. To improve the alignment, one way efficient is to combine this technique with other assembly techniques such as micro-fluidic assembly, contact printing, electric or magnetic field assembly. A further disadvantage is the reliance of this technique on the integrity of the biomolecules and biological environment used in the experiment, which increases the complexity and fragility of the assembly process.

\section{Molecular-Electrostatic-Interaction-Driven as- sembly}

Assembly of NW/NTs can also be driven by electrostatic interactions [38-47]. These interactions rely on either the inherent or induced polarity of these nanomaterials or a surface modification of these nanomaterials to adopt a specific charge. The working mechanism of electrostatic-actuated NWs assembly was shown in Fig. 1. When the patterned substrate is put in a NW solution, the electric interaction between the charged NWs and substrate leads the attachment and alignment of NWs on the charged regions of the substrate, as shown in Fig. 1 and Fig. 2.

For the charged substrate, one example is that silicon oxide obtains negative charge at $\mathrm{pH}=7$, which can attract NWs with positive charge. Silicon oxide surface can also be treated to carry positive charges. To fabricate specific NW patterns, the charged silica surface can be partially masked by the selective patterning with a dielectric layer, as shown in Fig. 1(a). Then the binding sites for NWs to reside on are pre-defined. If 
the binding site is narrow enough, it will be able to attract only single NWs, as shown in Fig. 2(d). And an important feature is that, the NWs are always adsorbed and focused onto the central area of the binding site (Fig. 2(d)).
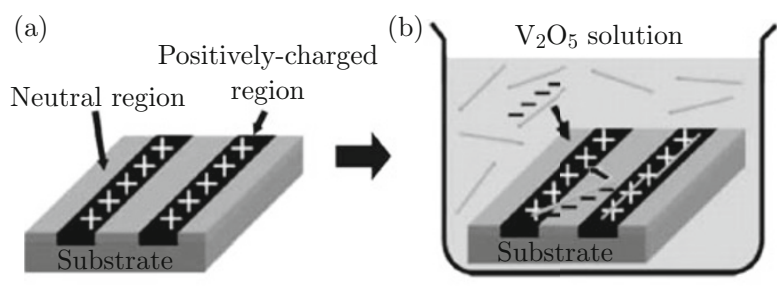

(d)

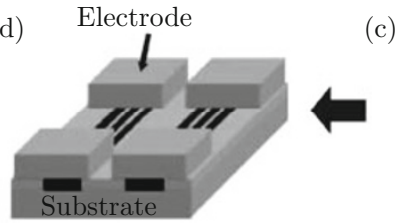

(c)

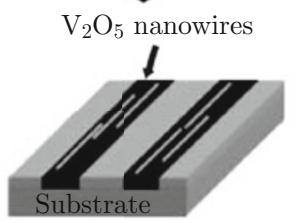

Fig. 1 Schematics of programmed electrostatic assembly of $\mathrm{V}_{2} \mathrm{O}_{5}$ NWs on substrates [38]. (a) Patterning self-assembled monolayers of either positively charged or neutral terminal groups. (b) Assembly and alignment of NWs directed by surface molecular pattern due to the charge. (c) Rinsing with DI water leaves aligned NW patterns on the substrate. (d) Subsequent microfabrication process to fabricate electrodes. (Reprinted with permission from [38]. (C) 2005 WILEY-VCH Verlag GmbH \& Co. KGaA).
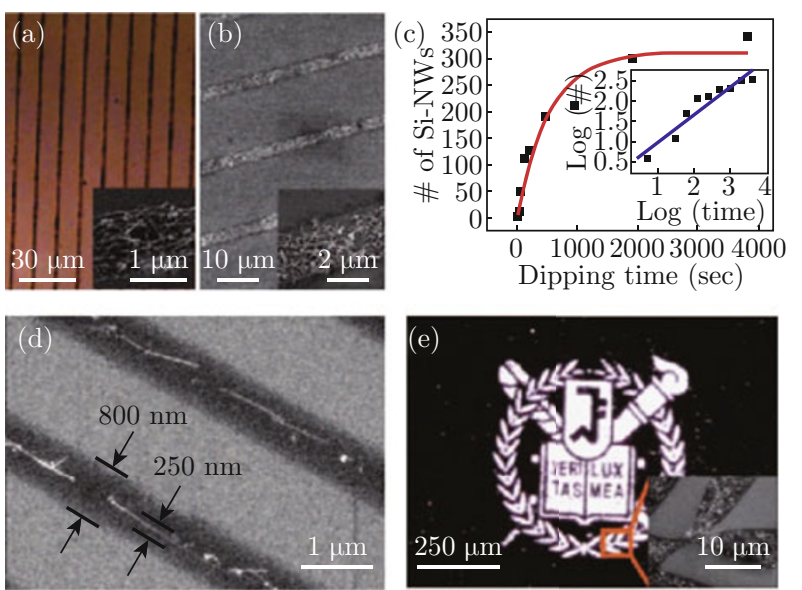

Fig. 2 Highly selective electrostatic assembly and alignment of SiNWs [39]. (a) NWs assembled on the Au substrates. (b) SiNWs assembled on $\mathrm{SiO}_{2}$ substrates. (c) Number of SiNWs adsorbed onto the substrate as a function of dipping time in the SiNW solution. (d) "Focused assembly" of functionalized SiNWs. (e) Functionalized SiNWs assembled onto complex patterns with arbitrary orientations. (Reprinted with permission from [39]. (C) 2008 American Chemical Society).

Large quantities of NWs can be assembled onto the substrate with the assistance of an electric field. As the dipping time in the NW solution increases for the substrate, the NWs attracted to corresponding binding sites increases, as summarized in Fig. 2(c). One typical advantage of this method is that it allows researchers to prepare arbitrary-shaped patterns of SiNWs using only conventional microfabrication facilities (Fig. 2(e)). A disadvantage is that the property of most NWs needs to be changed before being applicable in this technique. And this technique also has no control over the alignment of the NWs during the assembly process.

\section{Assembly within magnetic fields}

A magnetic field can direct the alignment of magnetic NWs suspended within a solution (Fig. 3). This technique has been limited to the assembly of NWs composed of ferromagnetic and/or superparamagnetic materials [48-55]. The process of assembly can be guided by external magnetic fields, as shown in Fig. 3(a), and the uniformity, alignment and positioning of the assembled patterns can be better controlled if magnets are precisely incorporated onto the substrate (e.g. attaching or patterning magnets onto the substrate).

NWs assembled onto these substrates will often form long chain-like structures extending from the magnet along its magnetic field lines, and those NWs are physically and probably electrically connected (Fig. 3(b)), which is very difficult or even impossible for other assembly techniques. Further demonstrations include the assembly of NWs into crossed-wire configurations and assembly into hierarchical structures via magnets positioned beneath the substrate (Fig. 3(c) and (d)). A major limitation of this technique is the material property that the NWs being assembled must respond to a magnetic field, greatly limiting eligible materials for this method. Future directions for this work could include the use of magnetic fluids to assist the assembly of non-magnetic NWs.

\section{Assembly within electric fields by dielec- trophoresis}

Electric field can direct the alignment of polarized NWs suspended within a solution (Fig. 4) by dielectrophoresis (DEP) [56-89]. It is an efficient process that can be applied to a number of NW materials (e.g., metals and semiconductors) and getting quite popular. Theoretical understanding was obtained on how materials behave in an applied electric field [56-58]. Briefly, the electric field induces charge separation (i.e. polarization) on the NWs, and the resulting polarization generates a dipole moment, which aligns the NWs to an energetically favorable orientation and location. DEP usually occurs at the periphery of the electrode fingers while depending on the field lines and their shape and the field strength (a minimum energy configuration within the applied field, i.e., parallel to the field lines), resulting in alignment of the NWs between two 


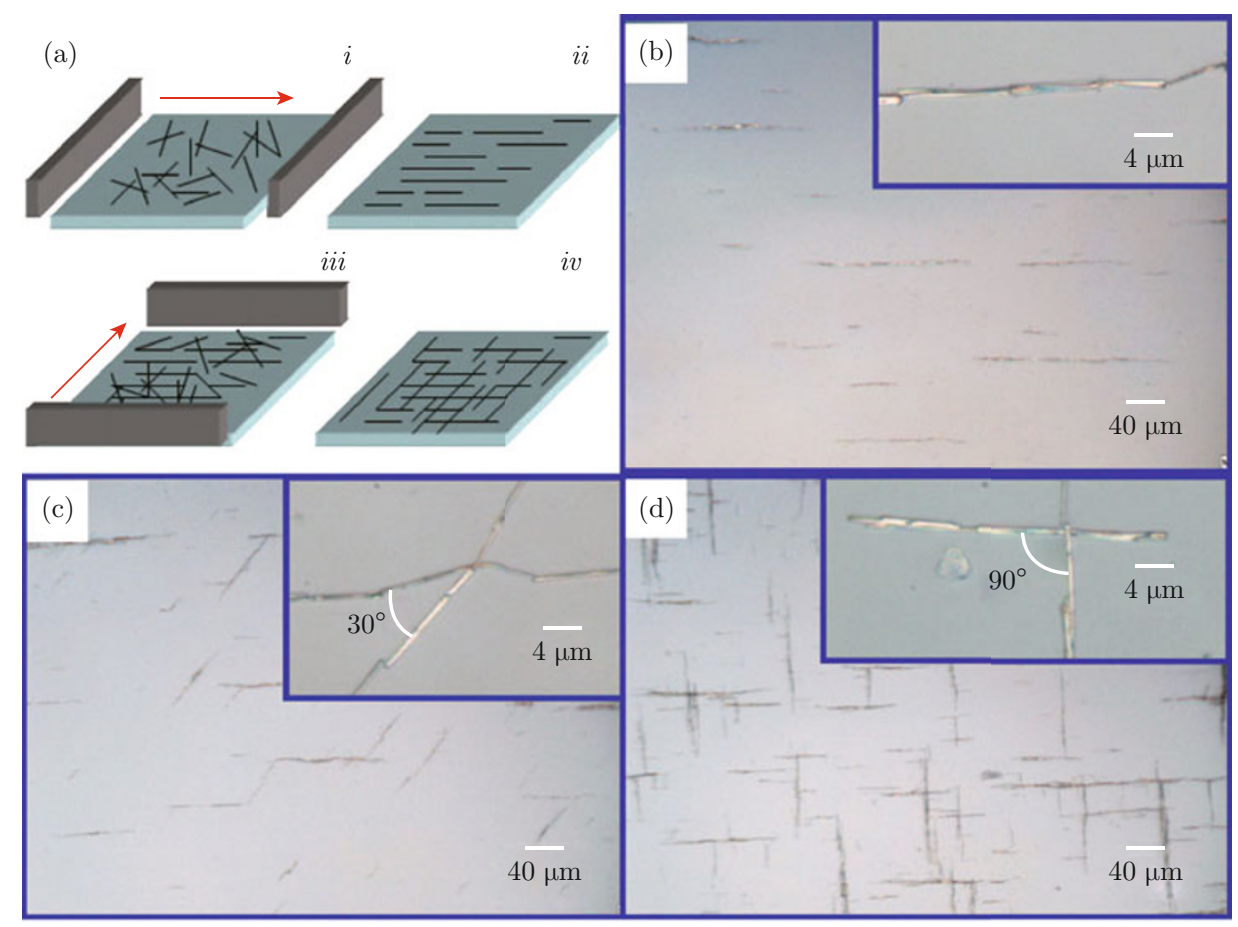

Fig. 3 Two-step process for sequential magnetic alignment [48]. (a) Schematics of the process of NWs aligning under an external magnetic field. (b) The alignment of the NWs in a defined direction. (c) A two-step alignment of crossed NWs with the substrate shift of $30^{\circ}$ between dispensing. (d) A two-step alignment with a substrate shift of $90^{\circ}$ between alignments. (Reprinted with permission from [48]. (C) 2007 IOP Publishing Ltd.).
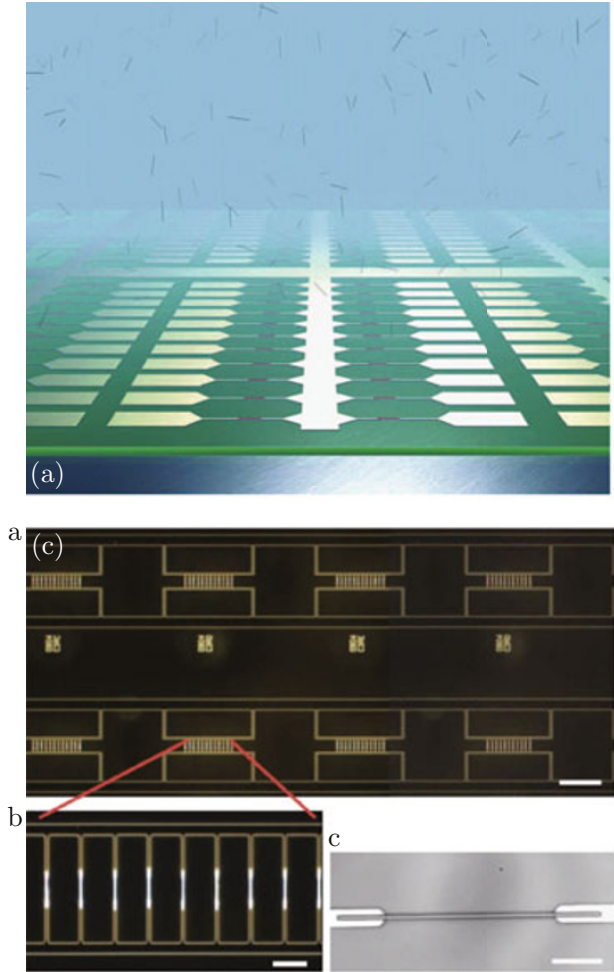
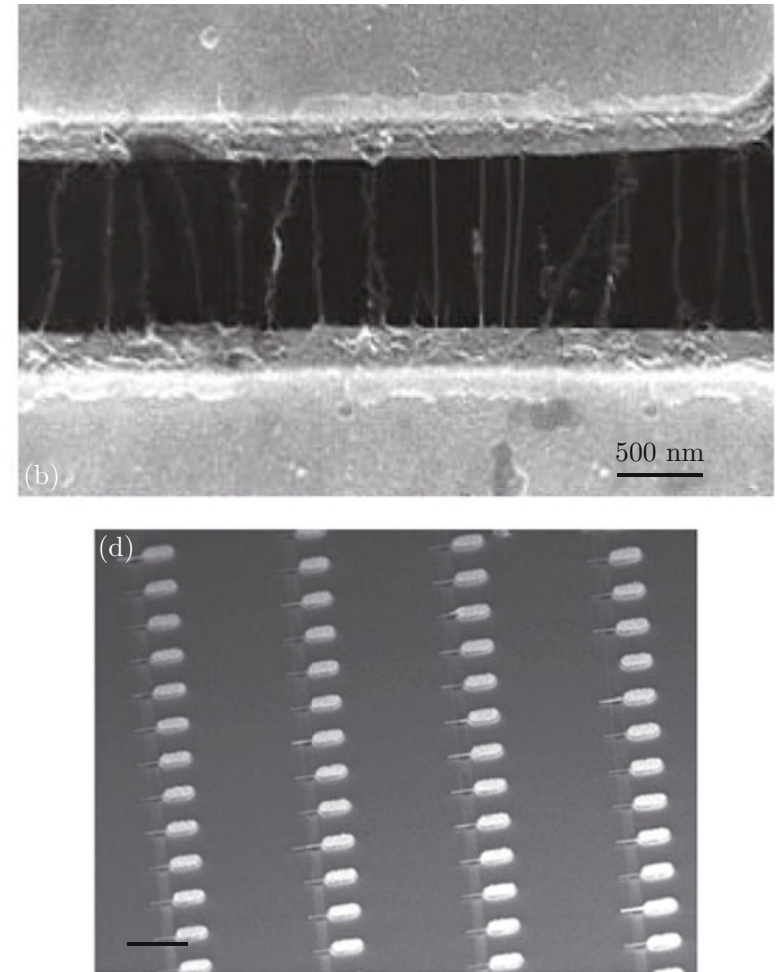

Fig. 4 NWs assembly by DEP. (a) Schematic of the high-density array of single-tube devices assembled by DEP [74]. (Reprinted with permission from [74]. (C) 2007 American Chemical Society). (b) Multiple SWCNTs aligned on the electrodes [71]. (Reprinted with permission from [71]. (C) 2006 IOP Publishing Ltd.). (c) Defect-free SiNWs assembled on eight multifinger electrode arrays, the three scale bars are $200 \mu \mathrm{m}, 20 \mu \mathrm{m}$ and $4 \mu \mathrm{m}$ separately [79]. (Reprinted with permission from [79]. (C) 2010 Nature Publishing Group). (d) RhNW resonator arrays of single NWs positioned at predefined locations on the chip, the scale bars is $20 \mu \mathrm{m}$ [81]. (Reprinted with permission from [81]. (C) 2008 Nature Publishing Group). 
electrode fingers, shown in Fig. 4(a). Although both $\mathrm{DC}$ and $\mathrm{AC}$ electric fields can be utilized to generate DEP, AC fields are more preferred in order to suppress undesired electrochemical interactions and/or reaction at the electrodes' surfaces, to reduce electrophoretic motion due to the NWs' electric charge. The alignment result depends on the electric voltage and frequency, as well as configurations and patterns of the electrodes.

NWs have been assembled using DEP in high concentrations [59-71] or as individual entities [59-61, 7274]. For example, C. Chen [71] fabricated and integrated single-wall carbon nanotubes(SWCNTs) using DEP assembly, as shown in Fig. 4(b), where multiple NWs are located over one pair of electrodes. By decreasing electrode size and picking NW concentration, applied voltage and frequency skillfully, E. M. Freer [79] and M. Li [81] assembled big amounts of NWs in a single-NW-electrode way where only one NW assemble on one electrode, as shown in Fig. 4(c) and (d).

The DEP-driven process is quite scalable and flexible, which can be implemented over multiple substrate sizes, and assembled NWs can be numerous as well as only one over a single pair of electrodes, based on DEP assembly's self-limiting features. This technique is so far the most reliable and controllable method for mass production of NW/NT based nanodevices. People may challenge for the necessity of patterning electrodes and applying voltage to direct the assembly process in this method, which may limit some of its applications However, if one can incorporate the purpose of electrodes for DEP with the circuit design of the final devices, there is no additional cost raised by this method. Potential problems include suspended NWs may form bundles either during or after DEP assembly. In addition, there is possibility that the electrodes and NWs may be burnt out when the gap between two electrodes is bridged and a large current pass through. However this may lead another interesting application of NW based devices, nano-fuses. NWs of low resistance can be integrated into nanodevices to provide overcurrent protection.

\section{Shear-Force-Driven NW alignment}

Shear force, associated with a flow and generated by the relative motion of a liquid and a solid object, can be the driving force for NW alignment. Flow can be achieved by a number of means, such as pressure, temperature difference, surface tension of capillary force etc. In these flows, shear force is parallel to the direction of flow movement. This force can redirect the orientation of NWs that are either suspended in a solution or attached to a surface. The new orientation of the majority of NWs is nearly in parallel to or within a small deviation of the direction of the shear force.

\section{Assembly by shear forces in a fluid}

Confining the flow to a microchannel or a pre-defined orientation by surface energy can establish a unidirectional shear force that can been used to align NWs (Fig. 5) [90-100]. The suspended NWs move in the flow direction and align along the direction of shear force. Some of them finally reside onto the surface and adopt the orientation parallel to the direction of shear force. For example, if the assembly process takes place in a solution in a microchannel, the density of NWs can be controlled by changing the concentration of NWs within the suspended liquid and flow duration, while the location and size of the assemble NWs are predefined by the microchannel. Repeating this process after realigning the microfluidic channel to other pre-defined directions can create layer-by-layer assemblies of NWs with desired patterns. Subsequent steps of NW deposition needs carefulness when realigning the microfluidic channel and the control of flow rates because which may reorient NWs deposited on the substrate during the previous steps of assembly processes. One approach combining fluid flow with hydrodynamic focusing $[99,100]$ is a recent advance in this method. With the help of the hydrodynamic focusing mechanism, the important NW array characteristics, such as its location, density, and dimension, can all be readily controlled by adjusting the flow rates of the sheath flows and the focusing flow. This method has no limit in the choice of NW materials.

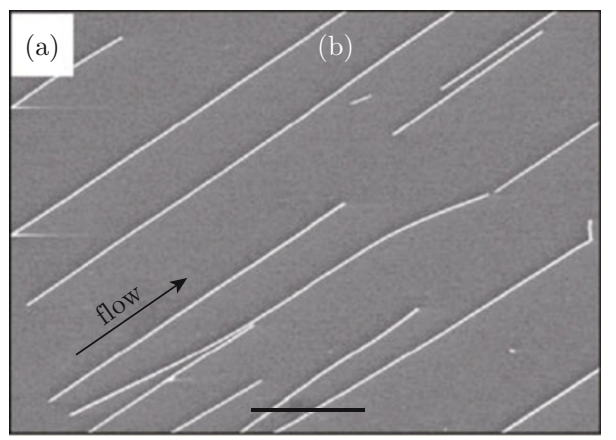

Fig. 5 Parallel assembly of InP NW arrays aligned by a channel flow. The scale bar is $2 \mu \mathrm{m}$ in the figure [90]. (Reprinted with permission from [90]. (C) 2001 American Association for the Advancement of Science).

The fluidic shear force driven assembly techniques are quite simple and economic. Future research may include controlling the NWs' location along the flow direction, by adding other available mechanisms.

\section{Assembly by Blown Bubble Films (BBF)}

In this approach, NWs are aligned at the interface between the NWs, the bubble film, and the substrate, not in a solution as most abovementioned methods [101,102]. The driving shear force, also a surface tension 
force, originates from liquid evaporation at the interface. The basic steps in the BBF approach (Fig. 6) are: (i) preparation of a polymer blend with homogeneous, stable and controlled concentration of NW suspension, (ii) blowing the polymer-nanowire mixture using a circular die to form a bubble with control of pressure and expansion rate, (iii) transfer of the bubble film onto a substrate for NW deposition. The NWs in the bubble film are all well-aligned, so they will remain aligned after assembly on the substrate. This approach can assemble NWs over large scales such as wafer scales, and the assembled NW density could be controlled by the NW concentration in the polymer suspension. It is simple, low-cost and versatile method that can be applied to nearly all types of substrates such as curved or soft substrate. However challenges remain for this technique in control of distribution and density of NWs on the bubble surface, the position of NWs to be deposited onto the substrate. In addition, a polymer coating may be introduced to the NW surface.
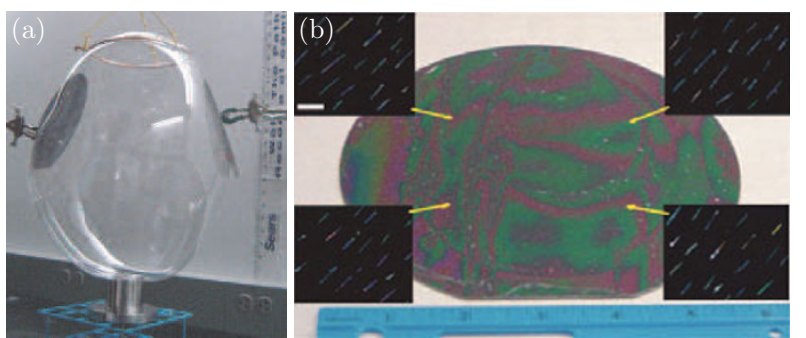

Fig. 6 (a) Directed bubble expansion process at its final stage, where the BBF has coated the substrate surface. (b) Image of SiNWs transferred onto a Si wafer. Insets are images showing SiNWs in the film. The scale bar is $10 \mu \mathrm{m}$, and is the same in all insets [101]. (Reprinted with permission from [101]. (C) 2007 Nature Publishing).

\section{Assembly by LB technique and LS technique}

Langmuir-Blodgett (LB) technique and LangmuirSchaefer (LS) techniques can also be applied to assemble high-density NW arrays [97,103-110], and they are also approaches that originates from interfacial shear forces, the same with the BBF method. In LB or LS approaches, NWs suspended at an air-liquid interface can be assembled into dense single-layer films through compression within an LB trough (Fig. 7(a)). During compression, the NWs within the thin film are closely packed in order to minimize the energy of the system. Dip coating is another means to transfer the NWs floating at the air-liquid interface onto a solid substrate (Fig. 7(b)). The NWs can adhere to the substrate through various interactions or their combination such as van der Waals, hydrophobic-hydrophilic, shear force or electrostatic interactions as the substrate is drawn in a vertical direction (LB technique) or in a horizontal direction (LS technique) from the layer of compressed NWs (Fig. 7(c)).
During the drawing process, as the solvent recedes from the surface of the substrate, a shear force is created along the air-liquid interface, leading to alignment of the NWs in the same direction, as shown in Fig. 8(a). The density of the deposited NWs is controlled by the speed of withdrawing the substrate from the liquid trough and the pressure applied to the assembly within the trough. The LB and LS techniques are quite scalable, which can be applicable to substrates with various sizes. In addition, with surface modification, these assembly processes by LB and LS technique can be applied to a wide variety of substrates of different surface properties. Multiscale or layer-by-layer structures can also be created by repeating the assembly process. For example, crossed NW layers can be obtained after changing the orientation of the substrate and repeating the NW transferring process, as shown in Fig. 8(b). Crossed NW arrays may be further created by photolithographically patterning and chemically removing the unwanted regions of NWs. The remaining NW arrays are ready for subsequent device fabrication, as shown in Fig. 8(c).

One of limitations of LB technique for NW assembly is reorganization of the NWs during dip coating that leads to overlapping features and gaps within the dense arrays of NWs. LS technique prevents the reorientation of NWs observed in LB technique, and its transferred arrays of NWs are more uniformly distributed across the receiving substrate [103]. However, in both methods, the NW density is hard to control.

\section{Assembly by contact printing}

The mechanical movement of two solid surfaces against each other (contact printing) can also generate a shear force, which can direct the assembly of vertically grown NWs into arrays with an orientation parallel to the direction of the applied force [111-119]. The contact printing is a 'dry' process, which is fit for dry-grown NWs and is totally different with abovementioned methods. In this process, NWs are firstly grown on a 'donor' substrate, then the donor substrate slides or rolls on top of a receiver chip coated with lithographically patterned resist, as shown in Fig. 9(a). In the process, when sliding the donor substrate on the receiver substrate, NWs are broken and laid down by the shear force, detached from the donor substrate and finally anchored on the receiver substrate by the van der Waals interactions, resulting in the direct transfer of aligned NWs to the receiver chip [111]. Later removal of precoated photoresist will enable the patterning of NWs, from very dense NW layers to detached NWs array, as shown in Fig. 9(b)-(c). NWs with an orthogonally crossed configuration can be achieved by assembling the NWs in two orthogonal directions (Fig. 9(d)-(e)) by repeating the contact printing process. 

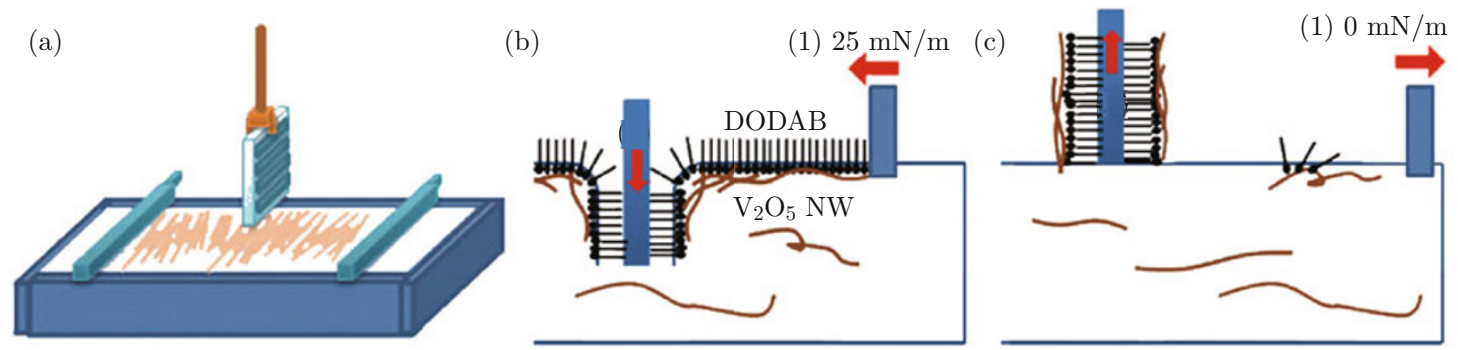

Fig. 7 Transfer process of the NW monolayer by the LB technique. (a) Formation of the NW LB film on the liquid surface by compressing the barrier. (b) Down-trip of a substrate through the NW film. (c) Opening the barrier to and lifting the substrate out of the liquid [103]. (Reprinted with permission from [103] (C) 2008 IOP Publishing Ltd.).
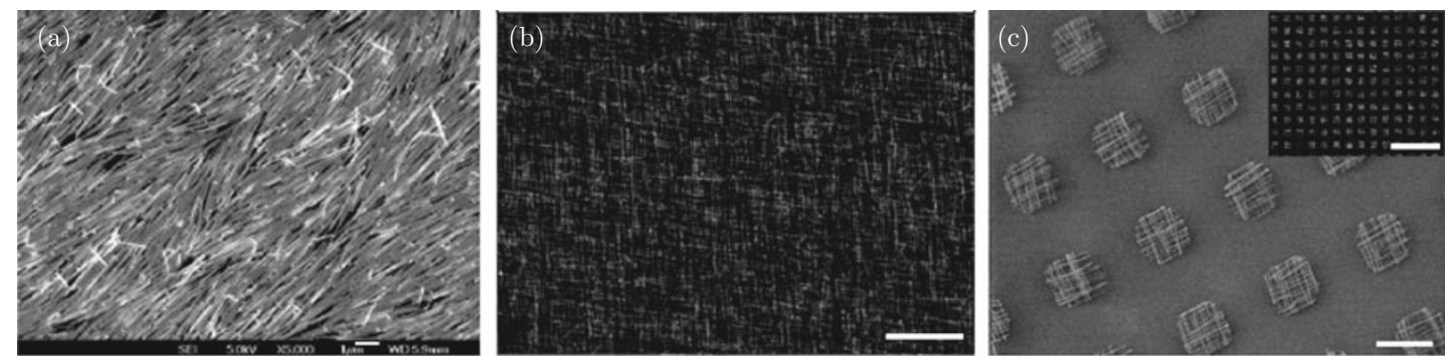

Fig. 8 (a) Silver NW monolayer deposited on a silicon wafer by LB technique [104]. (Reprinted with permission from [104]. (C) 2003 American Chemical Society). (b) Crossed SiNWs deposited uniformly on a substrate by repeating LB technique [105]. (Reprinted with permission from [105]. (C) 2003 American Chemical Society). (c) Patterned crossed SiNW arrays by photolithography [105]. The scale bars in the figures are respectively $1 \mu \mathrm{m}, 50 \mu \mathrm{m}$ and $10 \mu \mathrm{m}$. (Reprinted with permission from [105]. (C) 2003 American Chemical Society).
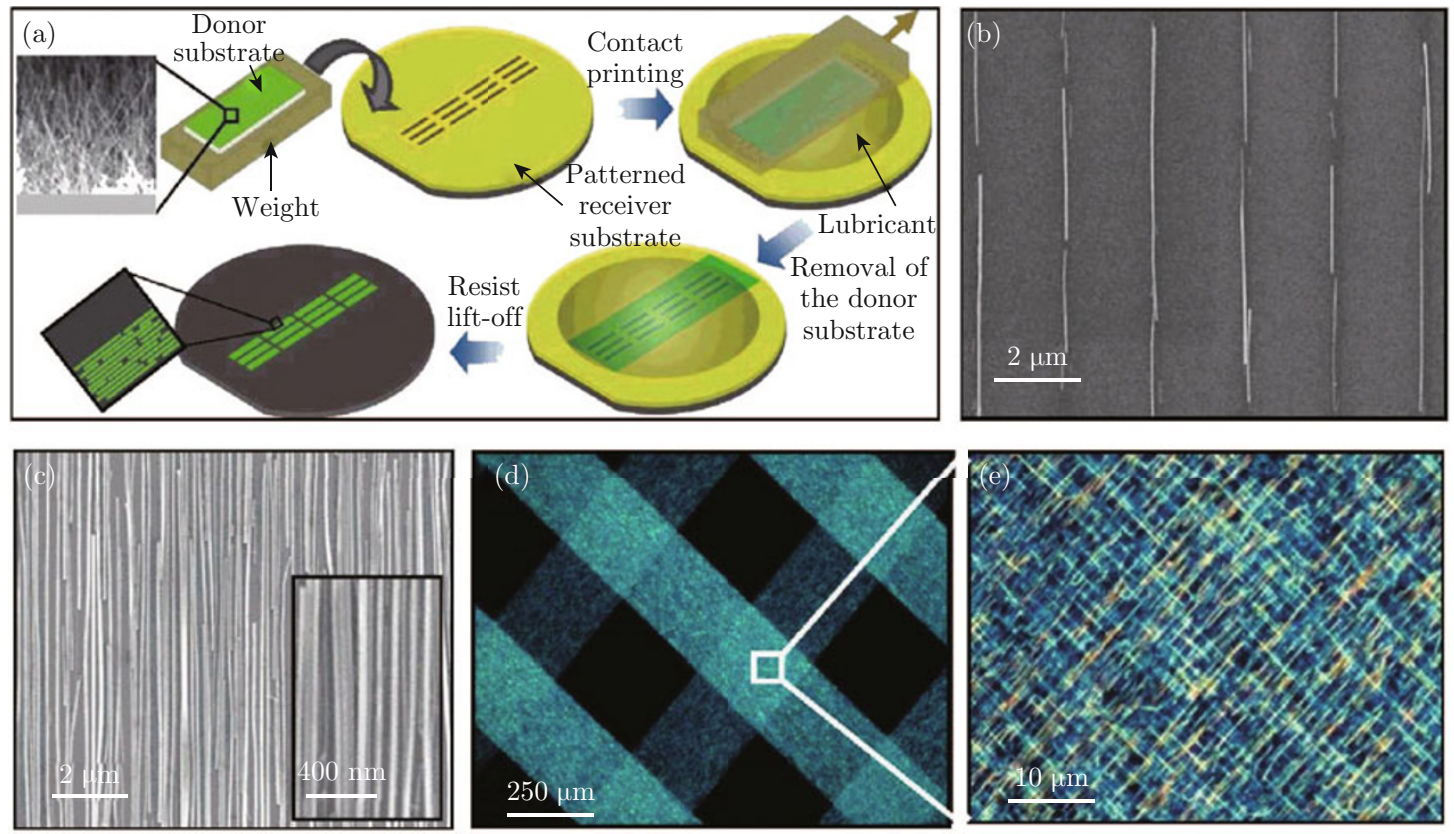

Fig. 9 (a) Schematic of the process flow for contact printing of NW arrays. (b) Regular array assembly of single GeNWs at predefined locations on a $\mathrm{Si} / \mathrm{SiO}_{2}$ substrate. (c) SEM images of GeNWs printed on a $\mathrm{Si} / \mathrm{SiO}_{2}$ substrate showing highly dense and aligned monolayer of NWs. (d) and (e) Optical images of double layer printing for SiNW cross assembly [111]. (Reprinted with permission from [111]. (C) 2008 American Chemical Society).

This assembly technique is feasible for dry-grown NWs, and can be scaled-up for high throughput. In this method, the pre-patterning of the receiver ensures that the position and density of assembled NW arrays can be precisely controlled, as shown in Fig. 9(b)-(c), which is very beneficial for later electrode registration and de- 
vice fabrication. A limitation is that, when transferring NWs from the donor substrate to the receiver substrate, NWs will break but the breaking point is random which results in a large variation of the length of NWs on the device substrate being transferred. And the balance of shear force and other interacting forces for transfer should be controlled tactically during the contact process.

\section{Assembly by knocking-down technique}

Knocking-down is also a 'dry' assembly process, and it can control the density, location, orientation and dimension of the NW array, which is very challenging for other techniques and makes it quite promising. The method, as depicted in Fig. 10, consists of two main steps, (i) an array of vertical SiNWs was firstly prepared by top-down DRIE (deep reactive ion etching) sculpting on SOI wafers. The vertical NW array was ordered and preprogrammed, with its location, density and dimension controlled. (ii) Followed is the 'knocking down' process, where the vertical NW array is knocked down by an appropriate elastomer-covered rigid-roller device [120].

This method is highly scalable, applicable to large size of substrates up to $10 \mathrm{~cm}^{2}$. Compared to the 'dry transfer or contact printing' approaches, the knockingdown method does not need a donor substrate, and there is no possibility of NWs broken during transferring. Thus the assembled NWs are highly uniform, which is very advantageous compared with other assembly methods. And this method is capable of controlling all possible array parameters, i.e., NW diameter and length, the number of NWs, NW density and orientation. And because of the highly controlled position and size of the NW elements in the method, contact electrodes can be easily created by lithography with a predefined mask with complete control over the number of NWs in each device and in a high yield production. Meanwhile in most abovementioned techniques, successful placement of NWs between the pre-fabricated or after-fabricated electrodes requires luck, which results in low reproducibility. The few disadvantages of this approach include that this method is limited to
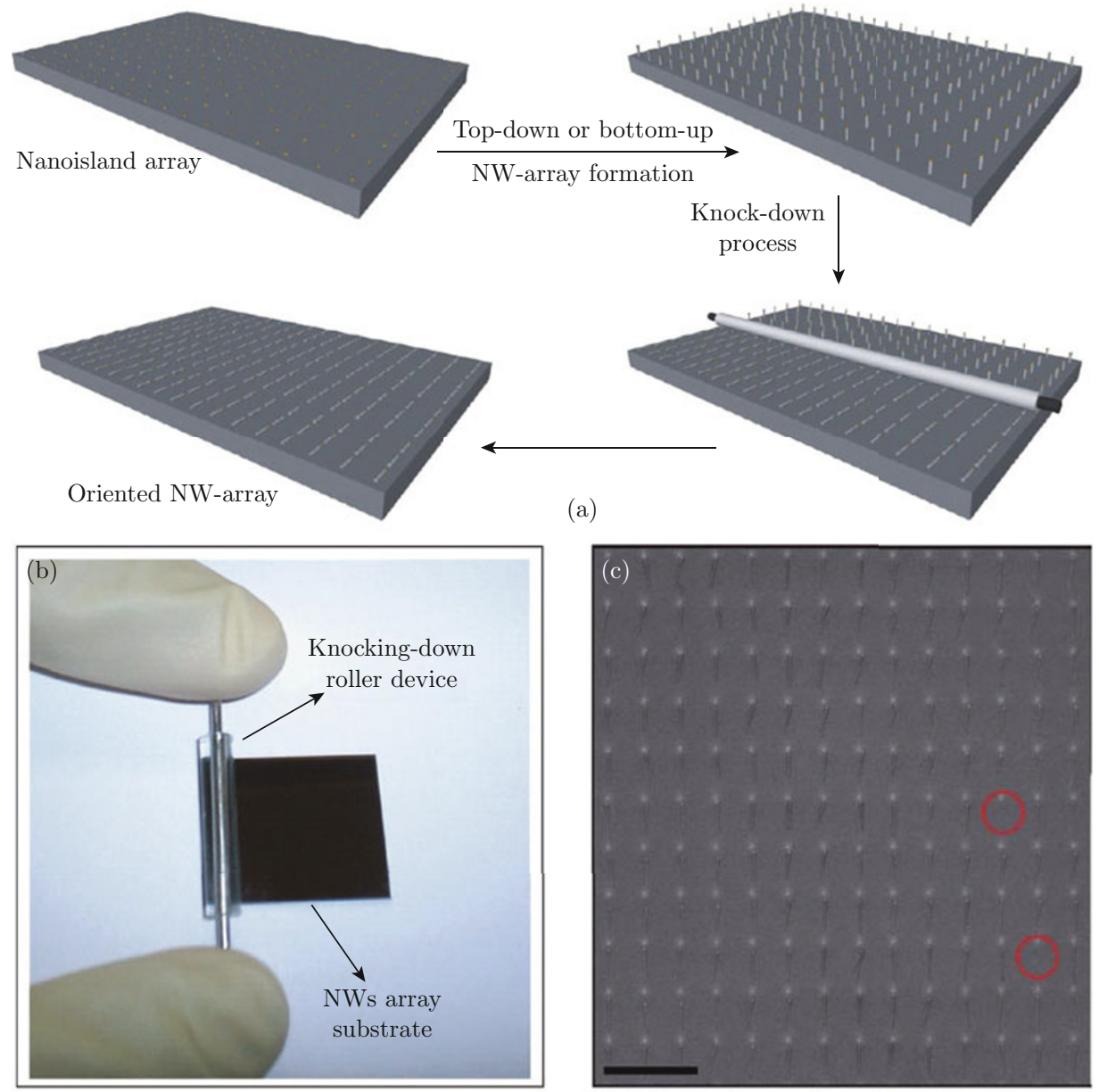

Fig. 10 (a) Schematic illustration of the knocking-down process for the fabrication of large-scale ordered NW arrays. (b) Hard-baked PDMS-covered roller element for the manual planarization of NW arrays. (c) A $15 \times 11 \mathrm{NW}$ array area after the knocking-down process. Red circles represent defective sites. Scale bar: $16 \mu \mathrm{m}$ [120]. (Reprinted with permission from [120]. (C) 2010 American Chemical Society). 

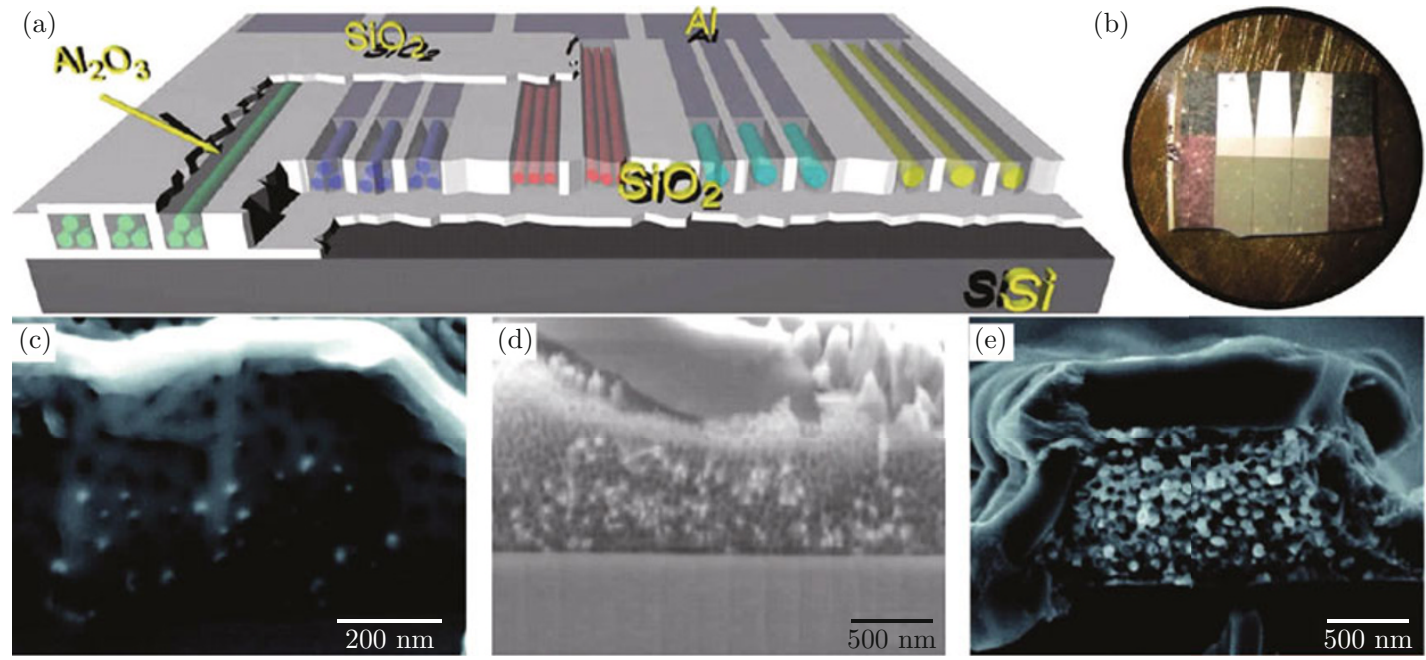

Fig. 11 Schematic of a multi-contact horizontal anodic aluminum oxide device. (b) The optical image of a representative three-contact device. The upper bright part is $\mathrm{Al}$, and the darker gray part is $\mathrm{SiO}_{2}$ layer. (c)-(e) SEM micrographs of electrodeposited NWs within the anodic alumina pore system: (c) gold NWs, (d) copper NWs, (e) TeNWs [121]. (Reprinted with permission from [121]. (C) 2010 American Chemical Society).

materials that is compatible with the nanofabrication process like the DRIE technique, or other NW materials that can be grown vertically, and this approach cannot assemble multiple types of NWs, or complex NW array configurations.

\section{Assembly of multiple types of NWs}

For many applications, for example, multibiomarker detection for biosensing, it is desirable to integrate different NW morphologies and types on a single chip. However, till now there is very little research on this topic. Y. Xiang reported a multi-contact design for the growth of multiple horizontal NW arrays [121]. Though not an Alignment-after-Growth approach, it is still worth mentioning since it may open up a new research interest for assembly of nanodevice.

In their design, individual contacts with various sizes are electrically separated from each other by physical distances, enabling their individual anodization. Thus, each contact would contain various nanopore morphologies, i.e., different pore diameters and different interpore distances, nanopore channels of different aspect ratios. Subsequently, the anodic pores were filled with various metals and semiconductors, such as gold, copper, cobalt, nickel and tellurium NWs, as shown in Fig. 11, thus a pattern including multiple types of NWs were created.

The multi-contact design established an effective and reproducible platform to synthesize multiple types of NWs, individually and independently addressable on a single chip. With semiconductor NWs, such as silicon or germanium, the multi-contact design offers the potential to create multiplex NW field-effect sensing devices. But this approach is quite complex and hard to control. And the NWs actually are grown at the edge of the chip, which are not easy to put into application.

Till now recent advances in technologies for assembling single or multiple types of NWs have been generally reviewed in this paper. It should be noted that although most of the techniques reviewed here are for assembling NWs, these methods can be generally applied to other one-dimensional nanomaterials, such as nanotubes, nanorods, and nanobelts. And whether an ohmic contact can be established between the NWs and later relating electrodes is also an important factor to judge the feasibility if assembly techniques. Every method has its own special characteristics, and to improve the alignment, different techniques may be combined together, such as electrostatic and magnetic field assembly [54], contact printing with strain force release on stretchable substrate [118-119].

\section{Conclusion}

Ultimately an ideal assembly process would be costeffective, reliable and able to direct the assembly of many NWs of different materials in parallel over a large area on a wide variety of substrates, as well as being able to manipulate and position single NWs of different materials, shapes and sizes. To select a feasible approach, one has to consider the specific application circumstances. Future research will focus on how to improve the process reproducibility and reliability, on the exploration of additional NWs with more compositions, on planar or non-planar plastic, metal or glass substrates, and also, the application of the ordered NW arrays in various electronic, optoelectronic and biosensing applications. 


\section{Acknowledgements}

The authors are grateful for the financial support from Natural Science and Engineering Research Council of Canada (NSERC), and funding from Science and Technology Commission of Shanghai Municipality (No. 11PJ1403500), the Open Project Program of State Key Laboratory of Industrial Control Technology (No. ICT1113) and Innovation Program of Shanghai Municipal Education Commission (No. 12YZ022).

\section{References}

[1] Y. Z. Long, M. Yu and B. Sun, Chem. Soc. Rev. 41, 4560 (2012). http://dx.doi.org/10.1039/ c2cs15335a

[2] X. Liu, Y. Z. Long and L. Liao, ACS Nano 6, 1888 (2012). http://dx.doi.org/10.1021/nn204848r

[3] M. C. P. Wang and B. D. Gates, Mater. Today 12, 34 (2009). http://dx.doi.org/10.1016/ S1369-7021 (09) 70158-0

[4] Z. Fan, J. C. Ho and T. Takahashi, Adv. Mater. 21, 3730 (2009). http://dx.doi.org/10.1002/adma. 200900860

[5] L. Huang, Z. Jia and S. O'Brien, J. Mater. Chem. 17, 3863 (2007). http://dx.doi.org/10.1039/b702080e

[6] B. Erdem Alaca, Inter. Mater. Rev. 54, 245 (2009). http://dx.doi.org/10.1179/174328009X411190

[7] Z. Liu, L. Jiao and Y. Yao, Adv. Mater. 22, 2285 (2010). http://dx.doi.org/10.1002/adma. 200904167

[8] X. Zhou, F. Boey and H. Zhang, Chem. Soc. Rev. 40, 5221 (2011). http://dx.doi.org/10.1039/ c1cs15045f

[9] Y. Ma, B. Wang and Y. Wu, Carbon 49, 4098 (2011). http://dx.doi.org/10.1016/j. carbon. 2011.06.068

[10] J. Chai, D. Wang and X. Fan, Nature Nanotechnol. 2, 500 (2007). http://dx.doi.org/10.1038/nnano. 2007.227

[11] B. Messer, J. H. Song and P. Yang, J. Am. Chem. Soc. 122, 10232 (2000). http://dx.doi.org/10.1021/ ja002553f

[12] Y. Shan and S. J. Fonash, ACS nano 2, 429 (2008). http://dx.doi.org/10.1021/nn700232q

[13] Y. Shan, A. K. Kalkan and C. Y. Peng, Nano Lett. 4, 2085 (2004). http://dx.doi.org/10.1021/nl048901j

[14] C. Cheng, R. K. Gonela and Q. Gu, Nano Lett. 5, 175 (2005). http://dx.doi.org/10.1021/nl048240q

[15] Y. Zhang, A. Chang and J. Cao, Appl. Phys. Lett. 79, 3155 (2001). http://dx.doi.org/10.1063/1.1415412

[16] S. Huang, X. Cai and J. Liu. J. Am. Chem. Soc. 125, 5636 (2003). http://dx.doi.org/10.1021/ja034475c

[17] X. Wang, Q. Li and G. Zheng, Nanotechnology 21,395602 (2010). http://dx.doi.org/10.1088/ 0957-4484/21/39/395602

[18] G. W. Slawinski and F. P. Zamborini, Langmuir 23, 10357 (2007). http://dx.doi.org/10.1021/ $1 \mathrm{a} 701606 \mathrm{p}$
[19] L. Ding, A. Tselev and J. Wang. Nano Lett. 9, 800 (2009). http://dx.doi.org/10.1021/n1803496s

[20] C. Kocabas, S. H. Hur and A. Gaur, Small 1, 1110 (2005). http://dx.doi.org/10.1002/smll. 200500120

[21] A. Rutkowska, D. Walker and S. Gorfman, J. Phys. Chem. C 113, 17087 (2009). http://dx.doi.org/10. 1021/jp9048555

[22] F. Rao, Y. Zhou and T. Li, Carbon 47, 2548 (2009). http://dx.doi.org/10.1016/j.carbon.2009.05.016

[23] X. Cao, B. Li and Y. Huang, ACS Appl. Mater. Interfaces 1, 1873 (2009). http://dx.doi.org/10.1021/ am900478y

[24] J. Xiao, S. Dunham and P. Liu, Nano Lett. 9, 4311 (2009). http://dx.doi.org/10.1021/n19025488

[25] J. Chen, F. Gao and L. Zhang, Mater. Lett. 63, 721 (2009). http://dx.doi.org/10.1016/j.matlet. 2008.12.036

[26] R. Cui, Y. Zhang and J. Wang, J. Phys. Chem. C 114, 15547 (2010). http://dx.doi.org/10.1021/ jp100286c

[27] I. Ibrahim, A. Bachmatiuk and F. Börrnert, Carbon 49, 5029 (2011). http://dx.doi.org/10.1016/j. carbon. 2011.07.020

[28] H. Wu, L. Chen and C. Tsai, Micro Nano Lett. 1, 25 (2006). http://dx.doi.org/10.1049/mnl:20065018

[29] H. Yan, L. Liu and Z. Zhang, Appl. Phys. Lett. 95, 143114 (2009). http://dx.doi.org/10.1063/1. 3242378

[30] G. H. Kim and W. D. Kim, Appl. Phys. Lett. 88, 233101 (2006). http://dx.doi.org/10.1063/1. 2210972

[31] M. B. Bazbouz and G. K. Stylios, J. Appl. Polym. Sci. 107, 3023 (2008). http://dx.doi.org/10.1002/app. 27407

[32] L. Xiao, Y. Zhang and Y. Wang, Nanotechnology 22, 025502 (2011). http://dx.doi.org/10.1088/ 0957-4484/22/2/025502

[33] K. Jiang, J. Wang and Q. Li, Adv. Mater. 23, 1154 (2011). http://dx.doi.org/10.1002/adma. 201003989

[34] J. K. N. Mbindyo, B. D. Reiss and B. R. Martin, Adv. Mater. 13, 249 (2001). http://dx.doi.org/10.1002/ 1521-4095 (200102) 13:4<249: : AID-ADMA249>3. 0 . CO ; $2-9$

[35] N. I. Kovtyukhova and T. E. Mallouk, Chem. Eur. J. 8, 4354 (2002). http://dx.doi.org/10.1002/ 1521-3765 (20021004) 8: 19<4354: : AID-CHEM4354>3. $0 . \mathrm{CO} ; 2-1$

[36] J. Lee, A. A. Wang and Y. Rheem, Electroanal. 19, 2287 (2007). http://dx.doi.org/10.1002/elan. 200704000

[37] M. Chen, L.Guo and R. Ravi, J. Phys. Chem. B 110, 211 (2006). http://dx.doi.org/10.1021/jp055204m

[38] S. Myung, M. Lee and G. T. Kim, Adv. Mater. 17, 2361 (2005). http://dx.doi.org/10.1002/adma. 200500682

[39] K. Heo, E. Cho and J. E. Yang, Nano Lett. 8, 4523 (2008). http://dx.doi.org/10.1021/n1802570m 
[40] J. Kang, S. Myung and B. Kim, Nanotechnology 19, 095303 (2008). http://dx.doi.org/10.1088/ 0957-4484/19/9/095303

[41] S. Myung, K. Heo and M. Lee, Nanotechnology 18, 205304 (2007). http://dx.doi.org/10.1088/ 0957-4484/18/20/205304

[42] S. Myung, J. Im and L. Huang, J. Phys. Chem. B 110, 10217 (2006). http://dx.doi.org/10.1021/ jp062108s

[43] Y. K. Kim and S. J. Park, Nanotechnology 18, 015304 (2007). http://dx.doi.org/10.1088/0957-4484/18/ 1/015304

[44] J. Im and J. Kang, J. Phys. Chem. B 110, 12839 (2006). http://dx.doi.org/10.1021/jp062146b

[45] R. S. Mclean, X. Huang and C. Khripin, Nano Lett. 6, 55 (2006). http://dx.doi.org/10.1021/n1051952b

[46] J. Im, L. Huang and J. Kang, J. Chem. Phys. 124, 224707 (2006). http://dx.doi.org/10.1063/1. 2206590

[47] M. Lee, J. Im and B. Lee, Nature Nanotechnol. 1, 66 (2006). http://dx.doi.org/10.1038/nnano.2006.46

[48] C. Hangarter, Y. Rheem and B. Yoo, Nanotechnology 18, 205305 (2007). http://dx.doi.org/10.1088/ 0957-4484/18/20/205305

[49] C. M. Hangarter and N. V. Myung, Chem. Mater. 17, 1320 (2005). http://dx.doi.org/10.1021/cm047955r

[50] M. Liu, J. Lagdani and H. Imrane, Appl. Phys. Lett. 90, 103105 (2007). http://dx.doi.org/10.1063/1. 2711522

[51] Y. Rheem, C. M. Hangarter and E. H. Yang, et al., IEEE Trans. Nanotechnol. 7, 251 (2008). http://dx. doi.org/10.1109/TNANO. 2008.917852

[52] C. Ooi and B. B. Yellen, Langmuir 24, 8514 (2008). http://dx.doi.org/10.1021/la801006g

[53] B. Yoo, Y. Rheem and W. P. Beyermann, et al., Nanotechnology 17, 2512 (2006). http://dx.doi.org/10. 1088/0957-4484/17/10/012

[54] M. G. Bellino, E. J. Calvo and G. J. Gordillo, Phys. Status Solidi RRL 3, 1 (2009). http://dx.doi.org/ 10.1002/pssr. 200802207

[55] J. Wang, L. Y. Zhang, P. Liu, T. M. Lan, J. Zhang, L. M. Wei, Eric Siu-Wai Kong, C.H. Jiang and Y. F. Zhang, Nano-Micro Lett. 2, 134 (2010). doi:10.5101/ $\mathrm{nml}$. v2i2.p134-138

[56] J. Boote and S. Evans, Nanotechnology 16, 1500 (2005). http://dx.doi.org/10.1088/0957-4484/16/ 9/015

[57] S. Evoy, N. DiLello and V. Deshpande, et al., Microelectron. Eng. 75, 31 (2004). http://dx.doi.org/10. $1016 / j$.mee. 2003.09.010

[58] Y. Liu, J. H. Chung and W. K. Liu, et al., J. Phys. Chem. B 110, 14098 (2006). http://dx.doi.org/10. 1021/jp061367e

[59] P. A. Smith, C. D. Nordquist and T. N. Jackson, et al., Appl. Phys. Lett. 77, 1399 (2000). http://dx.doi. org/10.1063/1.1290272

[60] D. Wang, R. Zhu and Z. Zhou, et al., Appl. Phys. Lett. 90, 103110 (2007). http://dx.doi.org/10.1063/1. 2711756
[61] X. Duan, Y. Huang and Y. Cui, et al., Nature 409, 66 (2001). http://dx.doi.org/10.1038/35051047

[62] O. Harnack, C. Pacholski and H. Weller, et al., Nano Lett. 3, 1097 (2003). http://dx.doi.org/10.1021/ nl034240z

[63] S. Lu, J. Chung and R. S. Ruoff, Nanotechnology 16,1765 (2005). http://dx.doi.org/10.1088/ 0957-4484/16/9/059

[64] A. Subramanian, B. Vikramadity and B. J. Nelson, et al., Proceedings of 12 th International Conference on Advanced Robotics 208 (2005). http://dx.doi.org/ 10.1109/ICAR. 2005.1507414

[65] K. Oh, J. H. Chung and J. J. Riley, et al., Langmuir 23, 11932 (2007). http://dx.doi.org/10.1021/ $1 \mathrm{a} 701755 \mathrm{~s}$

[66] J. Lee, K. Moon and M. Ham, et al., Solid State Commun. 148, 194 (2008). http://dx.doi.org/10.1016/ j.ssc. 2008.08 .022

[67] A. Motayed, M. He and A. V. Davydov, et al., J. Appl. Phys. 100, 114310 (2006). http://dx.doi.org/ 10.1063/1.2397383

[68] V. La Ferrara, B. Alfano and E. Massera, et al., IEEE Trans. Nanotechnol. 7, 776 (2008). http://dx.doi. org/10.1109/TNANO. 2008.926333

[69] A. O'Riordan, D. Iacopino and P. Lovera, et al., Nanotechnology 22, 105602 (2011). http://dx.doi.org/ 10.1088/0957-4484/22/10/105602

[70] X. Li, E. Chin and H. Sun, et al., Sens. Actuators B 148, 404 (2010). http://dx.doi.org/10.1016/j.snb. 2010.05 .062

[71] C. Chen and Y. Zhang, J. Phys. D: Appl. Phys. 39, 172 (2006). http://dx.doi.org/10.1088/0022-3727/ $39 / 1 / 025$

[72] H. W. Seo, C. S. Han and S. O. Hwang, et al., Nanotechnology 17, 3388 (2006). http://dx.doi.org/10. 1088/0957-4484/17/14/008

[73] A. I. Baca, J. J. Brown and K. A. Bertness, et al., Nanotechnology 23, 245301 (2012). http://dx.doi.org/ 10. 1088/0957-4484/23/24/245301

[74] A. Vijayaraghavan, S. Blatt and D. Weissenberger, et al., Nano Lett. 7, 1556-1560 (2007). http://dx.doi. org/10.1021/n10703727

[75] Z. Chen, Z. Wu and L. Tong, et al., Anal. Chem. 78, 8069-8075 (2006). http://dx.doi.org/10.1021/ ac0614487

[76] L. An and C. R. Friedrich, J. Appl. Phys., 105, 074314 (2009). http://dx.doi.org/10.1063/1.3093975

[77] C. Chen, W. Zhang and Y. Zhang, Appl. Phys. Lett. 95, 192110 (2009). http://dx.doi.org/10.1063/1. 3263723

[78] L. Liu, X. Ye and K. Wu, et al., Sensors 9, 1714 (2009). http://dx.doi.org/10.3390/s90301714

[79] E. M. Freer, O. Grachev and D. P. Stumbo, Nat. Nanotechnol. 5, 525 (2010). http://dx.doi.org/10.1038/ nnano. 2010.106

[80] S. Raychaudhuri, S. A. Dayeh and D. Wang, et al., Nano Lett. 9, 2260 (2009). http://dx.doi.org/10. 1021/n1900423g 
[81] M. Li, R. B. Bhiladvala and T. J. Morrow, et al., Nat. Nanotechnol. 3, 88 (2008). http://dx.doi.org/ $10.1038 /$ nnano. 2008.26

[82] N. Mohseni Kiasari and P. Servati, IEEE Electr. Device L. 32, 982 (2011). http://dx.doi.org/10.1109/ LED . 2011.2149492

[83] Z. Wang, M. Kroener and P. Woias, Sensor Actuat. A-Phys. (2012).

[84] D. A. Brown, J. H. Kim and H. B. Lee, et al., Sensors 12, 5725 (2012). http://dx.doi.org/10.3390/ s120505725

[85] L. Liao, J. Bai and R. Cheng, et al., Nano Lett. 12, 2653 (2011). http://dx.doi.org/10.1021/nl201922c

[86] S. J. Papadakis, J. A. Hoffmann and D. Deglau, et al., Nanoscale 3, 1059 (2011). http://dx.doi.org/10. 1039/c0nr00536c

[87] S. Y. Lee, A. Umar and D. I. Suh, et al., Physica E 40, 866 (2008). http://dx.doi.org/10.1016/j. physe.2007.10.094

[88] Z. Xiao and F. Camino, Nanotechnology 20, 135205 (2009). http://dx.doi.org/10.1088/0957-4484/20/ 13/135205

[89] P. Li and W. Xue, Nanoscale Res. Lett. 5, 1072 (2010). http://dx.doi.org/10.1007/s11671-010-9604-3

[90] Y. Huang, X. Duan and Q. Wei, et al., Science 291, 630 (2001). http://dx.doi.org/10.1126/ science.291.5504.630

[91] X. Duan, C. Niu and V. Sahi, et al., Nature 425, 274 (2003). http://dx.doi.org/10.1038/nature01996

[92] M. C. McAlpine, R. S. Friedman and S. Jin, et al., Nano Lett. 3, 1531 (2003). http://dx.doi.org/10. $1021 / \mathrm{n} 10346427$

[93] M. C. McAlpine, R. S. Friedman and C. M. Lieber, Nano Lett. 3, 443 (2003). http://dx.doi.org/10. 1021/nl034031e

[94] Y. Cui, Q. Wei and H. Park, et al., Science 293, 1289 (2001). http://dx.doi.org/10.1126/science. 1062711

[95] D. Wang, R. Tu and L. Zhang, et al., Angew. Chem. Int. Ed. 44, 2925 (2005). http://dx.doi.org/10. 1002/anie. 200500291

[96] S. Li, N. Liu and M. B. Chan-Park, et al., Nanotechnology 18, 455302 (2007). http://dx.doi.org/10.1088/ 0957-4484/18/45/455302

[97] D. Wang and H. Dai, Appl. Phys. A: Mater. 85, 217 (2006).

[98] Y. T. Liu, X. M. Xie and Y. F. Gao, et al., Mater. Lett. 61, 334 (2007). http://dx.doi.org/10.1016/j. matlet.2006.04.058

[99] M. Liu, Y. Chen and Q. Guo, et al., Nanotechnology 22, 125302 (2011). http://dx.doi.org/10.1088/ 0957-4484/22/12/125302

[100] M. Liu, Y. Chen and Q. Guo, et al., J. Nanoelectron. Optoelectron. 6, 144 (2011). http://dx.doi.org/10. 1166/jno. 2011.1153

[101] G. Yu, A. Cao and C. M. Lieber, Nat. Nanotechnol. 2, 372 (2007). http://dx.doi.org/10.1038/nnano. 2007.150
[102] G. Yu, X. Li and C. M. Lieber, et al., J. Mater. Chem. 18, 728 (2008).http://dx.doi.org/10.1039/ b713697h

[103] J. Park, G. Shin and J. S. Ha, Nanotechnology 19, 395303 (2008).http://dx.doi.org/10.1088/ 0957-4484/19/39/395303

[104] A. Tao, F. Kim, C. Hess, et al., Nano Lett. 3, 1229 (2003). http://dx.doi.org/10.1021/n10344209

[105] D. Whang, S. Jin and Y. Wu, et al., Nano Lett. 3, 1255 (2003).http://dx.doi.org/10.1021/n10345062

[106] S. Acharya, A. B. Panda and N. Belman, et al., Adv. Mater. 18, 210 (2006). http://dx.doi.org/10.1002/ adma. 200501234

[107] L. Jia, Y. Zhang and J. Li, et al., J. Appl. Phys. 104, 074318 (2008).http://dx.doi.org/10.1063/1. 2996033

[108] D. Wang, Y. L. Chang and Z. Liu, et al., J. Am. Chem. Soc. 127, 11871 (2005). http://dx.doi.org/ 10.1021/ja053836g

[109] S. Jin, D. Whang and M. C. McAlpine, et al., Nano Lett. 4, 915 (2004). http://dx.doi.org/10. $1021 / \mathrm{nl} 049659 \mathrm{j}$

[110] J. Li and Y. Zhang, Carbon 45, 493 (2007).http:// dx.doi.org/10.1016/j. carbon. 2006.10.027

[111] Z. Fan, J. C. Ho and Z. A. Jacobson, et al., Nano Lett. 8, 20 (2008). http://dx.doi.org/10.1021/nl071626r

[112] A. Javey, S. W. Nam and R. S. Friedman, et al., Nano Lett. 7, 773 (2007). http://dx.doi.org/10. $1021 /$ nl0630561

[113] R. Yerushalmi, Z. A. Jacobson and J. C. Ho, et al., Appl. Phys. Lett. 91, 203104 (2007). http://dx.doi. org/10.1063/1.2813618

[114] L. Jiao, B. Fan and X. Xian, et al., J. Am. Chem. Soc. 130, 12612 (2008). http://dx.doi.org/10.1021/ ja805070b

[115] Y. K. Chang and F. C. N. Hong, Nanotechnology 20, 195302 (2009).http://dx.doi.org/10.1088/ 0957-4484/20/19/195302

[116] L. Wen, K. M. Wong and Y. Fang, et al., J. Mater. Chem. 21, 7090 (2011). http://dx.doi.org/10.1039/ c1jm10496a

[117] J. Yang, M. S. Lee and H. J. Lee, et al., Appl. Phys. Lett. 98, 253106 (2011). http://dx.doi.org/ 10.1063/1.3601466

[118] G. W. Hsieh, J. J. Wang and K. Ogata, et al., J. Phys. Chem. C, 116, 7118 (2012).http://dx.doi.org/ 10.1021/jp210341g

[119] F. Xu, J. W. Durham and B. J. Wiley, et al., ACS nano 5, 1556 (2011).http://dx.doi.org/10. $1021 / \mathrm{nn} 103183 d$

[120] A. Pevzner, Y. Engel and R. Elnathan, et al., Nano Lett. 10, 1202 (2010).http://dx.doi.org/10.1021/ $\mathrm{nl903560u}$

[121] Y. Xiang, A. Keilbach and L. Moreno Codinachs, et al., Nano Lett. 10, 1341 (2010). http://dx.doi.org/ $10.1021 / \mathrm{n} 1904207 \mathrm{n}$ 